\title{
Neutron production by cosmic-ray muons at shallow depth
}

\author{
F. Boehm, ${ }^{3}$ J. Busenitz, ${ }^{1}$ B. Cook,${ }^{3}$ G. Gratta, ${ }^{4}$ H. Henrikson, ${ }^{3}$ J. Kornis, ${ }^{1}$ D. Lawrence, ${ }^{2}$ K. B. Lee, ${ }^{3}$ K. McKinny, ${ }^{1}$ \\ L. Miller, ${ }^{4}$ V. Novikov, ${ }^{3}$ A. Piepke, ${ }^{1,3}$ B. Ritchie, ${ }^{2}$ D. Tracy, ${ }^{4}$ P. Vogel, ${ }^{3}$ Y-F. Wang, ${ }^{4}$ and J. Wolf ${ }^{1}$ \\ (Palo Verde Collaboration) \\ ${ }^{1}$ Department of Physics and Astronomy, University of Alabama, Tuscaloosa, Alabama 35487 \\ ${ }^{2}$ Department of Physics and Astronomy, Arizona State University, Tempe, Arizona 85287 \\ ${ }^{3}$ Department of Physics, California Institute of Technology, Pasadena, California 91125 \\ ${ }^{4}$ Physics Department, Stanford University, Stanford, California 94305
}

(Received 23 June 2000; published 12 October 2000)

\begin{abstract}
The yield of neutrons produced by cosmic ray muons at a shallow depth of 32 meters of water equivalent has been measured. The Palo Verde neutrino detector, containing 11.3 tons of Gd loaded liquid scintillator and 3.5 tons of acrylic, served as a target. The rate of one and two neutron captures was determined. Modeling the neutron capture efficiency allowed us to deduce the total yield of neutrons, $Y_{\text {tot }}=(3.60 \pm 0.09 \pm 0.31) \times 10^{-5}$ neutrons per muon and $\mathrm{g} / \mathrm{cm}^{2}$. This yield is consistent with previous measurements at similar depths.
\end{abstract}

PACS number(s): 13.10.+q, 13.60.-r, 29.40.Mc, 98.70.Sa

\section{INTRODUCTION}

In the present work we report the results of a new measurement of muon-induced neutron production at shallow depth, based on the Palo Verde neutrino detector [1]. Even though the device was designed for a different purpose, namely the detection of reactor $\bar{\nu}_{e}$, it was possible to operate it in a mode suitable for the identification of muon-induced neutrons.

Neutrons and other hadrons produced by cosmic-ray muons in the Earth are an important and unavoidable source of background for underground low rate experiments. Knowledge of the rates for these processes is an essential part of understanding backgrounds in neutrino detectors and other low counting rate experiments. For example, since muon-produced fast single or multiple neutrons can mimic the correlated signature of inverse neutron beta decay, searches for neutrino oscillations at nuclear reactors $[1,2]$ must cope with this source of background. Other neutrino and proton decay experiments, as well as dark matter searches (even though often at greater depth), have to cope with this source of background as well. The CDMS experiment, for instance, is searching for cold dark matter [3], and is presently at shallow depth; muon-induced neutrons represent a major source of background. Low-energy accelerator neutrino oscillation searches $[4,5]$ are usually performed near the Earth's surface where muon-induced neutrons are a significant source of background. Another example is the proposed ORLaND neutrino detector at the Spallation Neutron Source at Oak Ridge National Laboratory [6] which is also planned to be at shallow depth.

Despite the importance of the subject, relatively little recent progress has been reported. Several measurements of the neutron production rates at various depths conducted in the past suggest a smooth dependence of the neutron yield on depth or, equivalently, on the average muon energy [7-9]. However, a more recent measurement by the LVD Collaboration [10], resulting in a smaller yield, is in disagreement with this simple dependence on depth. At shallow depth, where the average muon energy is $\sim 10 \mathrm{GeV}$, the smooth trend was confirmed by measurements with relatively small detectors without much shielding against the neutrons produced outside them $[7,11]$. In the more recent experiment [11], the single and double neutron yields were determined separately, and pion production by muons was also observed. One drawback of that experiment, possibly present in the other ones as well, is the difficulty of distinguishing between neutrons produced in the detector (the intended source of neutrons) and neutrons produced outside by the muoninduced showers. In fact, in Ref. [11] it has been estimated that half of the detected single neutrons originated in hadron showers outside the detector volume.

The theoretical description of this background process is usually based on the assumption that the electromagnetic interaction of high-energy muons with matter can be modeled by replacing the exchanged virtual photon by "equivalent" real photons and using known photo-nuclear reaction cross sections [12]. The analysis is complicated, since in order to relate the theoretical neutron production yield to measurement, the propagation and possible cascade multiplication of all reaction products must be understood. [For example, a $\pi^{-}$produced by a muon will make more neutrons, as does the $(n, 2 n)$ reaction, etc.] While the smooth variation noted above for the neutron reaction rate versus depth is supported in some calculations [13], other approaches come to different conclusions [14,15]. In particular, Ref. [15] was devoted, unlike the others, to the relatively shallow depth relevant for the present work. In that work, the calculated neutron yield was smaller than the measured one [11], while the calculated and measured $\pi^{+}$yields agreed with each other. One should also keep in mind that while the theoretical description quoted above deals with the muon-nucleus interaction involving the exchange of a virtual photon, neutrons can be also produced by nuclear interactions involving real bremsstrahlung photons, and electron-positron pairs created during the passage of muons through matter. (The present experiment cannot separate neutrons created by interactions involving virtual photons from those produced by interactions of real photons.) 


\section{EXPERIMENT}

The measurement of the neutron production rate was performed at $32 \pm 3$ meter-water-equivalent (mwe) depth using the Palo Verde neutrino detector. The detector and its operation is described in detail in Ref. [1]. Briefly, the apparatus consists of 66 acrylic cells, each $900 \times 12.7 \times 25.4 \mathrm{~cm}^{3}$. These cells are filled with liquid scintillator loaded with $\mathrm{Gd} 0.1 \%$ by weight, for a total scintillator volume of 11.34 tons. The acrylic material has an aggregate mass of 3.48 tons. Muons can thus spall either in the scintillator volume or in the acrylic material, and the resulting neutrons cannot be distinguished by source. Neutrons, when moderated to thermal energies, are preferentially captured on Gd, resulting in a $\sim 8$ $\mathrm{MeV}$ gamma-ray cascade, the characteristic neutron capture signal. The central active detector volume is surrounded by a 1-m-thick water shield, and the outermost layer of the detector is an active muon veto counter providing $4 \pi$ coverage.

Since the apparatus was intended for the detection of reactor neutrinos, it was not optimized for the neutron yield measurement. For the present purpose special runs were performed without the trigger rejection of the cosmic rays. The throughgoing muon sample was selected off line such that at least two veto hits were recorded. Only muons which at the same time went through at least three cells of the central detector were included. The delayed neutron capture events, recognized by their energy deposit, are of two kinds: The "single bank" events, where only one neutron capture candidate event occurs following the veto hit, and the "two bank" events, which have two neutron capture candidate events following the muon. (The detector electronics is not capable of recording more than two correlated events.)

The measured quantities are the numbers of single $\left(N_{1}\right)$ and double neutron-capture events $\left(N_{2}\right)$ associated with $N_{\mu}$ muons traversing the central detector. The average path length of these muons in the central detector is $X$ (measured in units of $\mathrm{g} / \mathrm{cm}^{2}$ ). Since some of the neutrons could have been created in the water shield or in other external detector parts, correction factors $Q_{k}<1$ are applied to $N_{k}, k=1,2$. Finally, the neutron detection efficiencies $\epsilon_{k, l}$ are introduced. Here, e.g., $\epsilon_{1,1}$ is the probability that one neutron was created and one detected, while $\epsilon_{1,2}$ is the probability that two neutrons were created and only one detected. If all these quantities were known, one could define neutron yields $Y_{l}$, etc. (per muon and $\mathrm{g} / \mathrm{cm}^{2}$ ), where $l$ is the number of neutrons, which are independent of the detector properties and obey the relation

$$
N_{k}=\frac{N_{\mu} X \epsilon_{k}^{d a q}}{Q_{k}} \cdot \sum_{l=1}^{3} Y_{l} \epsilon_{k, l} .
$$

Here $\epsilon_{k}^{d a q}$ is the detection livetime correction which in this case depends on the number of detected neutron-like events $k$.

Obviously, since only two quantities, $N_{1}$ and $N_{2}$, are measured, only two yields can be determined, and the system above cannot be solved without approximations. It is assumed further that the contributions from four or more pro- duced neutrons can be neglected, as indicated in Eq. (1). Moreover, as will be shown below, the total neutron yield

$$
Y_{t o t}=Y_{1}+2 Y_{2}+3 Y_{3}
$$

is essentially independent of the ratio $Y_{3} / Y_{2}$, i.e., on the assumed value of $Y_{3}$, while the deduced single and double neutron yields $Y_{1}$ and $Y_{2}$ depend on that ratio significantly. Thus, the final results of the present experiment will be expressed as the measurement of $Y_{t o t}$.

Note that the quantities $Y_{l}$, and thus also $Y_{t o t}$, contain all processes that lead to the production of $l$ neutrons by the muon. Thus, if the muon creates a $\pi^{-}$(or any other particle except a neutron) which then, in turn, creates $k$ neutrons, all of the neutrons, regardless of source, contribute to $Y_{l}$. This somewhat awkward definition is necessary since the evaluation of the efficiencies $\epsilon_{k, l}$ is based on a code that tracks just neutron propagation in the detector.

The neutron capture events were recorded in two runs, each about half of a day long. The raw muon veto rate was about $2 \mathrm{kHz}$ and the rate of muons which went through at least three cells of the central detector and caused two or more detectable veto hits in the first (second) run was 270 (275) Hz. These two runs are essentially equivalent, and the final total number of muons was determined to be $N_{\mu}$ $=1.42 \times 10^{7}$, very similar in both runs.

The average path length $X$ of the muons is estimated with a simple ray-tracing Monte Carlo simulation, starting from a $\cos ^{2} \theta$ zenith angle distribution. (On the flat terrain at the shallow depth of $\sim 30$ mwe the $\cos ^{2} \theta$ was checked to be adequate. Moreover, the value of $X$ is not very sensitive on the assumed angular distribution.) The resulting $X$ was 125 $\mathrm{g} / \mathrm{cm}^{2}$ for the central detector [i.e., the scintillator, the acrylic cells and the small amount of other materials $(\mathrm{Cu}, \mathrm{Fe})$ in the central detector] and 317 and $62 \mathrm{~g} / \mathrm{cm}^{2}$ for the water shield and veto counter, respectively.

The neutron capture events $N_{k}$ were selected using cuts similar to the neutron part of the neutrino signal [1]. For single neutron events a time cut of 10-100 $\mu$ s between the last muon event and the neutron capture event was used, while for two neutron events only the lower limit of $10 \mu \mathrm{s}$ was kept, the upper one being irrelevant. The time between the two neutron capture events was restricted to 5-100 $\mu$ s. The energy distributions of all neutron events had identical shapes within statistics.

There are several contributions to the live time correction factors $\epsilon_{k}^{d a q}$. The largest one, which affects primarily the single neutron events, arises since the initial information is overwritten when another muon strikes the veto after the first neutron event was recorded and before the end of the $450 \mu \mathrm{s}$ time interval during which the data acquisition system (DAQ) waits for the second neutron event. Combining the correction for this effect with other dead time corrections, the resulting $\epsilon_{1}^{d a q}=30.8 \%(27.8) \%$ for one-neutron events in the first (second) runs, and $\epsilon_{2}^{d a q}=72.4 \% \quad(70.3 \%)$ for twoneutron events. The difference in $\epsilon_{k}^{d a q}$ between the two runs is caused primarily by the $\sim 8 \%$ change in the raw muon rate.

The distribution of the time interval between the muon and the single-neutron event is shown in the top panel of Fig. 

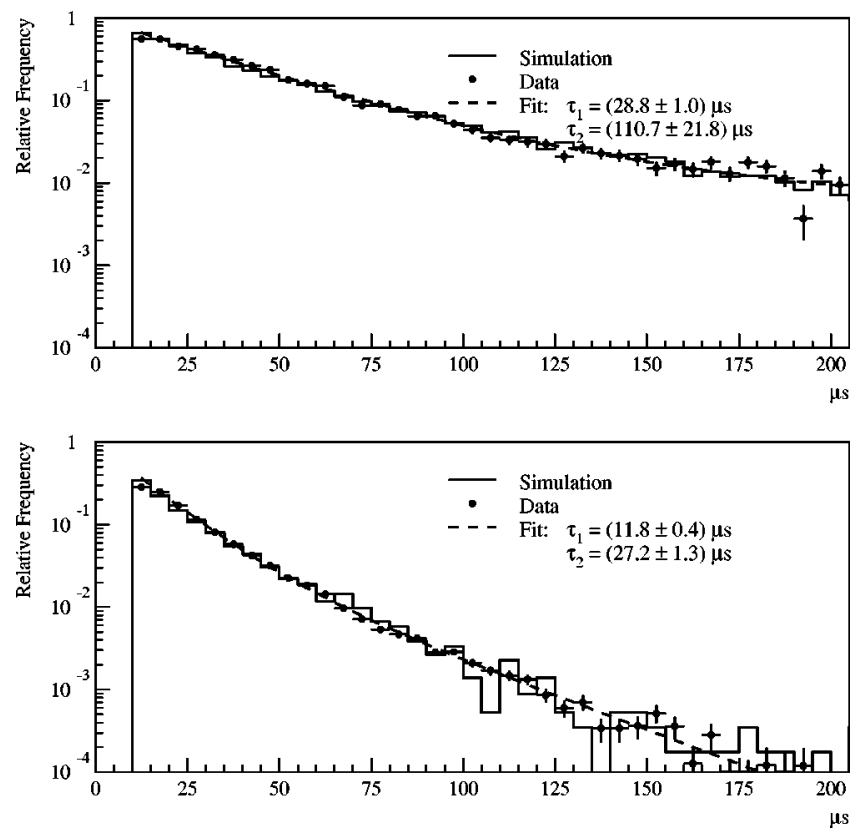

FIG. 1. Top: the distribution of time between muon hits and the single-neutron capture events, fitted to the exponential function, Eq. (3). Bottom: the distribution of time between muon hits and the first neutron capture events for the double-neutron events with an exponential fit, Eq. (3). In both panels the Monte Carlo simulation is shown as a histogram, and the fitted time constants $\tau_{1}$ and $\tau_{2}$ are displayed.

1, while the lower panel shows the same distribution for the first of the two neutron events. The fitted curves are of the form

$$
F(t)=\left(a_{1} e^{-t / \tau_{1}}+a_{2} e^{-t / \tau_{2}}+a_{3}\right) e^{-t / 500 \mu \mathrm{s}} .
$$

Here $\tau_{1}=28.8 \pm 1.0 \mu$ s for the single neutron case while $\tau_{1}$ $=11.8 \pm 0.4 \mu \mathrm{s}$ (not very far from half of the previous $\tau_{1}$, the value one expects for two neutrons) for the double neutron case are the characteristic neutron capture times. Both agree quite well with the Monte Carlo (MC) simulation, as seen in Fig. 1. The presence of the time constant $\tau_{2}$ is related to the inhomogeneous nature of the detector. Some neutrons enter the acrylic, where there is no Gd available for captures, thus prolonging their capture time. The smaller value of $\tau_{2}$ in the lower panel (double-neutron events) reflects the overall reduction of the capture process in the multineutron events. Finally, the last term, with a fixed time constant of $500 \mu \mathrm{s}$, represents the accidental background (500 $\mu$ s is the average time interval between successive muons). Using such a fit one can subtract the accidental background in the relevant time window, and calculate the corresponding correction for the described choice of the time cuts.

In Fig. 2 the distribution of the elapsed time between the two neutron capture events is shown, and again fitted to the same functional dependence, Eq. (3). (The distinction between the top and bottom panels is explained below.) Here, the time constant $\tau_{1}=18.1 \pm 1.0 \mu \mathrm{s}$ in the top panel is substantially smaller than the $\tau_{1}$ obtained by the MC simulation $\left(\tau_{1}^{M C} \sim 29 \mu \mathrm{s}\right.$, as expected if only two neutrons are in-
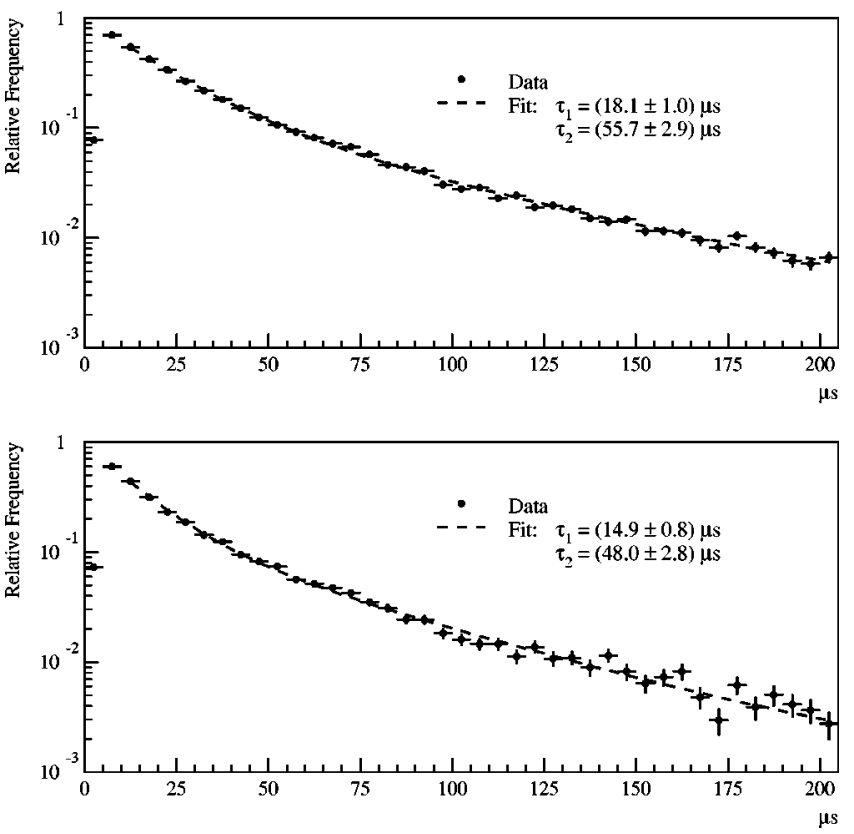

FIG. 2. Top: the distribution of the elapsed time between the two capture events with the two-veto-hit pattern for the doubleneutron events with an exponential fit, Eq. (3). Bottom: the distribution of the elapsed time between the two capture events for the subset of the double-neutron events with three veto hits. In both panels the fitted time constants $\tau_{1}$ and $\tau_{2}$ are displayed.

volved). This feature suggests that the data sample contains a non-negligible component with more than two neutrons. (Again, $\tau_{2}$ is noticeably smaller in the lower panel, for the same reasons as in Fig. 1.)

\section{EFFICIENCIES}

While the evaluation of $N_{\mu}, N_{1}, N_{2}$, and $X$ is rather straightforward, the determination of the efficiency matrix $\epsilon_{k, l}$ is somewhat model dependent. This is because, in order to calculate $\epsilon_{k, l}$, one needs the initial energy and angular distributions of the created neutrons, both of which are poorly known. In practice, a number of physically plausible assumptions are made about these distributions, and the spread among the resulting efficiencies yields a measure of the systematic uncertainty. Once the neutron initial distribution is chosen, the Monte Carlo simulation of the neutron transport developed for the neutrino experiment [1] is used. The detector geometry, materials and electromagnetic interactions are simulated using GEANT [16]. Hadronic interactions are simulated by GFLUKA [17] and the low energy neutron transport by GCALOR [18].

To evaluate the quantities $\epsilon_{1,1}$ and $\boldsymbol{\epsilon}_{2,1}$ initial single neutrons were distributed randomly in position and initial angles through the detector volume. For the initial energy distribution, several possibilities were used: the exponential distribution $\exp (-E / 39 \mathrm{MeV})$ as proposed by the Karmen Collaboration [19] and functions with the power dependence $E^{-x}$, $0.5 \leqslant x \leqslant 2$ (see [20]). These cover the shape following $E^{-1.86}$ suggested by experience with photo-nuclear processes [21]. All of these distributions result in similar efficiencies. Using 
the spread of the calculated values as a measure of the systematic error, and taking a simple average as the most probable value, one obtains

$$
\epsilon_{1,1}=0.20 \pm 0.03, \quad \epsilon_{2,1}=0.007 \pm 0.004
$$

Note that the probability that a single initial neutron will result in two neutron captures, characterized by $\epsilon_{2,1}$, is quite small.

The determination of two neutron efficiencies is even more difficult, since it depends on the energy distribution of both initial neutrons. To evaluate the quantities $\epsilon_{1,2}$ and $\epsilon_{2,2}$ two neutrons were created at the same random point and with random directions for each of them. Two extremes were considered. In the first, the total energy of the neutrons followed one of the previously described functions used above in the single neutron case. This initial energy was distributed randomly between them. (Essentially the same efficiency was obtained if the two neutrons shared the initial available energy equally.) The other extreme is obtained if both neutrons each have the energy distribution used in the single neutron case; i.e., the total neutron energy is on average twice as large. The spread (not very large) was again used in the averaging and in the estimate of the systematic error, resulting in

$$
\epsilon_{1,2}=0.22 \pm 0.01, \quad \epsilon_{2,2}=0.06 \pm 0.01
$$

Note that there is a sizable probability that only one neutron is detected when two were initially created. None of the previous analyses, in particular Ref. [11], took that into account. Next, one has to consider the possibility that three neutrons were spalled by the muon, but only one or two were detected. Again, using the average and the two extreme possibilities to divide the available energy among the three neutrons, one obtains

$$
\epsilon_{1,3}=0.19 \pm 0.01, \quad \epsilon_{2,3}=0.10 \pm 0.01
$$

It is important to realize that these efficiencies are not much smaller than those for the initial one or two neutrons. Thus, the effect of $Y_{3}$ should be considered. (The effect of four and more neutron spallations will be neglected, however.)

The correction factors $Q_{k}$ that exclude neutrons created outside the central detector volume (i.e. in the water shield since the effect of the veto is negligible), but captured there, must be determined also. To do that, the efficiencies for each passive volume were determined using the same Monte Carlo code as for the case of the central detector. Then $Q_{k}$ were determined from

$$
Q_{k}=\frac{\left(X \sum_{l} Y_{l} \epsilon_{k, l}\right)_{\text {central det }}+\left(X \sum_{l} Y_{l} \epsilon_{k, l}\right)_{\text {water }}}{\left(X \sum_{l} Y_{l} \epsilon_{k, l}\right)_{\text {central det }}},
$$

resulting in $Q_{1}=0.80 \pm 0.10$ and $Q_{2}=0.94 \pm 0.07$. In order to obtain the above values a crude assumption $Y_{1}=2 Y_{2}=2 Y_{3}$, was used. It is important to note that the uncertainties of $Q_{k}$ are strongly correlated with the error in the efficiencies.

The effect of the neutron component of hadronic cascades created outside the veto, and coincident with the muon which created them, remains to be determined. The ratio of muons making three hits in the veto, thus involving more than one particle, to the prevalent case of the two hits was employed as a measure of the frequency of showers. This ratio was $6.7 \pm 0.3 \%$ and $7.3 \pm 0.3 \%$ in the first and second runs, respectively, i.e., about $7 \%$ of muons entering the veto were accompanied by a shower.

To see that the "triple-veto-hit" events are really different from the standard throughgoing muons with just two veto hits, one can form ratios $R_{k}$,

$$
R_{k}=\frac{N_{k}^{\text {shower }} / N_{k}^{\text {total }}}{N_{\mu}^{\text {shower }} / N_{\mu}^{\text {total }}},
$$

which represent a quantitative measure of the neutron content of the showers. The measured values are $R_{1}=2.0 \pm 0.1$ and $R_{2}=4.4 \pm 0.2$, both significantly larger than unity. Thus the shower events indeed are richer in neutrons, particularly in the two neutron sample.

Moreover, the double neutron events with three veto hits have distinctly different time structure than the more common ones with just two veto hits. This is shown in the bottom part of Fig. 2 for the interevent time, i.e. the elapsed time between the first and second neutron captures. The corresponding time constant $\tau_{1}$ for the three-veto-hit events is significantly smaller than for the two-veto-hit events, showing that the shower events have a large multineutron component. A similar effect is present when the capture time of the first neutron is considered. It is therefore likely that the discrepancies between data and simulation in the time dependence of the interevent time interval, noted above, are the consequence of a multineutron $(l>2)$ component in the two bank events.

Since the shower events contain an unknown number of neutrons created outside the detector volume, they are excluded from further consideration.

\section{RESULTS}

In the first run, the observed numbers of neutron captures, corrected for the random background and with the effect of showers subtracted, were $N_{1}=3916 \pm 66, \quad N_{2}=828 \pm 29$, while in the second run $N_{1}=3451 \pm 62, N_{2}=829 \pm 29$. The two runs, which were separated in time by 10 months, give consistent results when the differences in $\epsilon^{d a q}$ is taken into account, proving that the experiment is stable.

As a first step, the yields are analyzed as in Ref. [11]; i.e., only the "diagonal" efficiencies $\epsilon_{k, k}$ are taken into account. Thus

$$
Y_{k}^{\text {simple }}=N_{k} Q_{k} /\left(N_{\mu} X \epsilon_{k, k} \epsilon_{k}^{d a q}\right) .
$$

The resulting yields obtained by averaging the two runs are 
TABLE I. Values of the neutron yields $Y_{\text {tot }}, Y_{1}, Y_{2}$, and $Y_{3}$ in units of $10^{-5}$ neutrons $/\left(\mu \mathrm{g} \mathrm{cm}^{-2}\right)$ for different assumed ratios $Y_{3} / Y_{2}$.

\begin{tabular}{rcccc}
\hline \hline Assumed $Y_{3} / Y_{2}$ & $Y_{\text {tot }}$ & $Y_{1}$ & $Y_{2}$ & $Y_{3}$ \\
\hline 0. & 3.54 & 2.30 & 0.62 & 0.0 \\
0.5 & 3.60 & 2.48 & 0.32 & 0.16 \\
1. & 3.62 & 2.54 & 0.22 & 0.22 \\
2. & 3.64 & 2.59 & 0.13 & 0.26 \\
\hline \hline
\end{tabular}

$$
\begin{aligned}
& Y_{1}^{\text {simple }}=[2.94 \pm 0.04(\text { stat }) \pm 0.50(\text { syst })) \times 10^{-5} \frac{\text { neutrons }}{\mu \mathrm{g} \mathrm{cm}^{-2}} \\
& Y_{2}^{\text {simple }}=[0.98 \pm 0.03(\text { stat }) \pm 0.14(\text { syst })] \times 10^{-5} \frac{\text { neutrons }}{\mu \mathrm{g} \mathrm{cm}^{-2}} .
\end{aligned}
$$

The exclusion of external showers, characterized by the three-veto-hit events, has resulted in the reduction of $Y_{1}^{\text {simple }}$ by $14 \%$ and of $Y_{2}^{\text {simple }}$ by $45 \%$. Thus, presumably due to the presence of the sizable shield $X \simeq 380 \mathrm{~g} / \mathrm{cm}^{2}$, the effect of the external showers, while still clearly present, was reduced compared to the findings of Ref. [11], where (in the same units) $\quad Y_{1}^{\text {simple }}=4.3(2.0) \times 10^{-5} \quad$ and $\quad Y_{2}^{\text {simple }}=1.6(0.5)$ $\times 10^{-5}$ without (with) the correction for external showers.

However, the proper analysis should include the full efficiency matrix, i.e., the possibility that two neutrons were initially produced but only one neutron capture was recorded and vice versa, as well as the possibility that three neutrons were originally produced and only two or one neutron captures were observed. As pointed out earlier, with only two measured capture rates, $N_{1}$ and $N_{2}$, it is impossible to deduce all the relevant information without further constraints. To avoid these difficulties, and to make the comparison with other experiments easier, the total number of neutrons produced per muon was evaluated. This quantity, $Y_{\text {tot }}$, defined earlier in Eq. (2), has the further advantage that it is essentially independent of the ratio $Y_{3} / Y_{2}$ for three to two neutron production, as demonstrated in Table I.

Thus, the final result of the present measurement can be expressed as

$$
Y_{\text {tot }}=(3.60 \pm 0.09 \pm 0.31) \times 10^{-5} \frac{\text { neutrons }}{\mu \mathrm{g} \mathrm{cm}^{-2}}
$$

where the systematic error is an estimate based on the spread of values in Table I added in quadrature to the spread of the evaluated efficiencies.

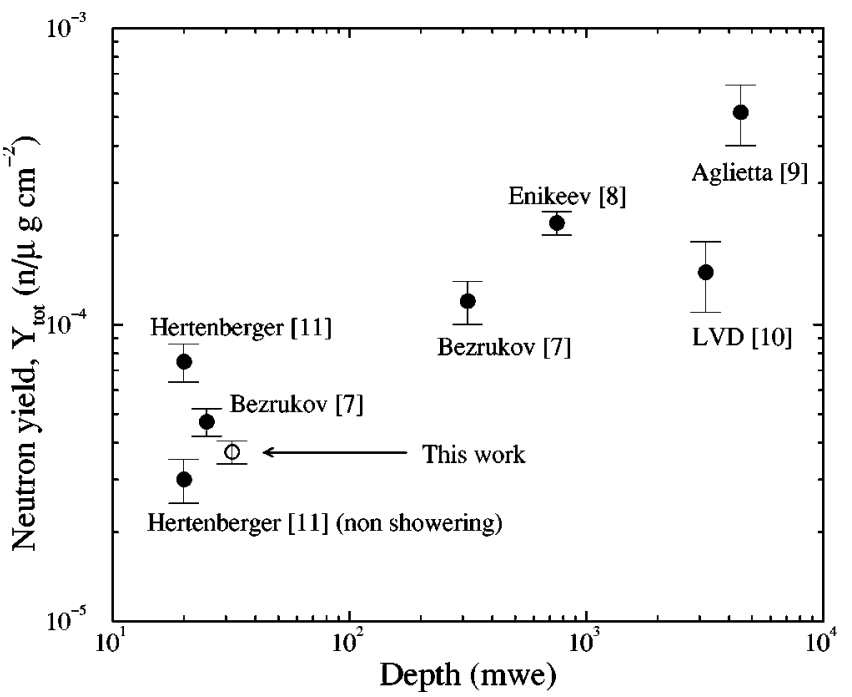

FIG. 3. Summary of available data on the total neutron yield $Y_{\text {tot }}$ versus depth. The points are labeled by the corresponding first author. For Ref. [11] two results are shown, with and without the effect of external showers. Present work is denoted by an open circle.

All available data on neutron yields are collected and compared in Fig. 3. (It is assumed that the other measurements [7-10] also are really $Y_{\text {tot }}$ measurements.) For the measurements of Ref. [11] both results, with and without the correction for external showers, are shown. In some of the other measurements the shower contribution was excluded, but it is not clear how well this has been done, since at least the results at 25 and 316 mwe were obtained with relatively small and unshielded detectors.

At shallow depth there are now three measurements, with essentially consistent results. Clearly, still better and more complete measurements are desirable, in which the full neutron multiplicity and energy spectra are determined and the neutrons produced externally are reliably identified.

\section{ACKNOWLEDGMENTS}

We would like to thank the Arizona Public Service Company for the generous hospitality provided at the Palo Verde plant. The important contributions of M. Chen, R. Hertenberger, K. Lou, and N. Mascarenhas in the early stages of this project are gratefully acknowledged. We also acknowledge generous financial help from the University of Alabama, Arizona State University, California Institute of Technology, and Stanford University. Finally, our gratitude goes to CERN, DESY, Fermilab, LANL, LLNL, SLAC, and TJNAF who at different times provided us with parts and equipment needed for the experiment. This project was supported in part by the U.S. DOE.
[1] F. Boehm et al., Phys. Rev. Lett. 84, 3764 (2000); F. Boehm et al., Phys. Rev. D 62, 072002 (2000).

[2] M. Apollonio et al., Phys. Lett. B 466, 415 (1999).

[3] R. Abusaidi et al., Nucl. Instrum. Methods Phys. Res. A 444,
345 (2000).

[4] C. Athanossopoulos et al., Phys. Rev. Lett. 81, 1774 (1998), and references therein.

[5] K. Eitel, Nucl. Phys. B (Proc. Suppl.) 70, 210 (1999), and 
references therein.

[6] B. D. Anderson et al. (unpublished).

[7] L. B. Bezrukov et al., Yad. Fiz. 17, 98 (1973) [Sov. J. Nucl. Phys. 17, 51 (1973)].

[8] R. I. Enikeev et al., Yad. Fiz. 46, 1492 (1987) [Sov. J. Nucl. Phys. 46, 883 (1987)].

[9] M. Aglietta et al., Nuovo Cimento C 12, 467 (1987).

[10] M. Aglietta et al., hep-ex/9905047.

[11] R. Hertenberger, M. Chen, and B. L. Dougherty, Phys. Rev. C 52, 3449 (1995).

[12] C. F. Weizsäcker, Z. Phys. 88, 612 (1934); E. J. Williams, K. Dan. Vidensk. Selsk. Mat. Fys. Medd. 13, 4 (1935).

[13] G. T. Zatsepin and O. G. Ryazhskaya, Izv. Akad. Nauk SSSR, Ser. Fiz. 29, 1946 (1965); L. B. Bezrukov and E. V. Bugaev, Yad. Fiz. 33, 1195 (1981) [Sov. J. Nucl. Phys. 33, 635 (1982)].

[14] O. C. Alkofer and R. D. Andersen, Nucl. Phys. B8, 402
(1968).

[15] J. Delorme et al., Phys. Rev. C 52, 2222 (1995).

[16] R. Brun et al., computer code GEANT 3, Report No. CERN DD/EE/84-1 (revised), 1987.

[17] A. Fasso et al., in Proceedings of the IV International Conference on Calorimetry in High Energy Physics, edited by A. Menzione and A. Seribano (World Scientific, Singapore, 1993), p. 493.

[18] T. A. Gabriel et al., Report No. ORNL/TM-5619-mc, 1977.

[19] Karmen Collaboration, B. Armbruster (private communication).

[20] J. C. Barton, in Proceedings of the 19th International Conference on Cosmic Rays, La Jolla, 1985 (Institute of Physics, London, 1985), p. 98.

[21] F. F. Khalchukov et al., Nuovo Cimento C 6, 320 (1983). 